\title{
Reflexiones en torno a las competencias y competencias tecnológicas en la formación superior en Bolivia
}

\author{
Reflexions about competencies and technological competencies in Bolivian higher \\ education
}

\section{Reflexões sobre competências e competências tecnológicas no ensino superior na Bolívia}

\author{
Mercedes Chamoso Luna \\ mercedeschamoso@gmail.com \\ https://orcid.org/0000-0002-7066-6903
}

Universidad Mayor, Real y Pontificia de San Francisco Xavier de Chuquisaca, Chuquisaca-Bolivia

Recibido 14 de mayo 2021 | Arbitrado y aceptado 1 de junio 2021 | Publicado en 01 julio 2021

\section{RESUMEN}

El siguiente artículo tiene por objetivo preponderar la importancia del modelo de formación por competencias $y$, por otro lado, el desarrollo de las competencias tecnológicas dentro de la educación superior donde las TIC juegan un rol preponderante desde su incorporación en el ámbito educativo. Desde aquí que el estudio se ha centrado en la realización de una revisión sistemática de la literatura y para organizar la información por categorías se utilizó el método cualitativo de análisis de contenido, para ello, se establecieron los insumos teóricos y aspectos centrales en torno a dos elementos: las competencias y las competencias tecnológicas en el ámbito educativo, así como la importancia del desarrollo de las mismas en un contexto laboral cada vez más competitivo y cambiante. Los resultados arrojan la visión que deben tener los futuros docentes sobre el desarrollo de las competencias y las competencias tecnológicas. Por lo que se concluye que ambos elementos al ser polisémicos contribuyen en el mejoramiento de la calidad educativa dentro de la formación superior.

Palabras clave: Competencias; competencias tecnológicas; educación superior

\section{ABSTRACT}

The following article aims to highlight the importance of the competencybased training model and, on the other hand, the development of technological competencies in higher education where ICTs play a predominant role since their incorporation into the educational environment. From here, the study has focused on the realization of a systematic review of the literature and to organize the information by categories, the qualitative method of content analysis was used, for this, the theoretical inputs and central aspects were established around two elements: competencies and technological competencies in the educational field, as well as the importance of their development in an increasingly competitive and changing labor context. The results show the vision that future teachers should have about the development of competencies and technological competencies. Therefore, it is concluded that both elements, being polysemic, contribute to the improvement of educational quality in higher education.

Key words: Competencies; technological competencies; higher education

\section{RESUMO}

O artigo seguinte visa destacar a importância do modelo de treinamento baseado em competências e, por outro lado, o desenvolvimento de competências tecnológicas no ensino superior, onde as TIC desempenham um papel predominante desde sua incorporação ao ambiente educacional. A partir daí o estudo concentrou-se na realização de uma revisão sistemática da literatura e para organizar as informações por categorias, foi utilizado o método qualitativo de análise de conteúdo, para o qual foram estabelecidos os inputs teóricos e os aspectos centrais em torno de dois elementos: competências e competências tecnológicas na esfera educacional, bem como a importância de desenvolvê-las em um contexto de trabalho cada vez mais competitivo e mutável. Os resultados mostram a visão que os futuros professores devem ter sobre o desenvolvimento de competências e competências tecnológicas. Conclui-se, portanto, que ambos os elementos, sendo polissêmicos, contribuem para a melhoria da qualidade da educação no ensino superior.

Palavras-chave: Competências; competências tecnológicas; ensino superior 


\section{INTRODUCCIÓN}

Las universidades de manera particular buscan hoy en día ser el centro del pensamiento, cultura e innovación, para lo cual asumen los nuevos enfoques universales de mayor tendencia que tiene como norte, la formación de profesionales competentes con conocimientos, habilidades y actitudes; en función a lo señalado, algunos estudios señalan al aprendizaje y la formación por competencias como uno delos enfoques educativos quefacilitan en mayor grado la transferencia de los aprendizajes (Boahin, 2014 y Hofman; Grossman y Salas, 2011, citados en: Rodríguez y Armengol, 2017. Para Camperos Camero (2008), hoy en día lo que la sociedad exige son instituciones educativas que formen en los años de estudio personas competentes; esto condiciona a que se deben formar profesionales vinculados con el campo profesional o de desempeño social, siendo que la sola ejercitación académica y la adquisición de conocimientos no es una garantia del dominio y pericia de las competencias profesionales, menos aún si la formación se la hace por asignaturas o disciplinas aisladas sin ningún hilo integrador que permita aprehender la complejidad e interrelaciones de funciones y sistemas propios para el ejercicio profesional.

Esta situación conlleva a que las universidades tanto europeas como latinoamericanas vengan asumiendo dentro de sus políticas educativas la formación por competencias, con la finalidad de responder a las exigencias sociales de contar con profesionales idóneos y competentes, capaces de vincularse con su medio y transformar el mismo.

Asimismo, según Camperos Camero (2008) señala que las competencias son asumidas en la educación hace más de tres décadas, lo hicieron en Estados Unidos, su expansión tiene 20 años, y se generalizó con la aplicación hecha en el Reino Unido, con la formulación de normas para su calificación, bajo un enfoque próximo al conductismo. El propósito del enfoque basado en competencias
(EBC) era el de sintonizar los programas de pregrado universitario con las necesidades del medio, resulta que dicha sintonización se dio a su vez con el sector productivo nacional y la inserción internacional, es a partir de esta visión holística e integral del proceso educativo que se impacta en el rol que ejerce tanto el docente como el estudiante (Corvalán, 2008). En este sentido dicho enfoque busca facilitar que los estudiantes adquieran los contenidos de los diferentes programas de asignatura a través de una manera más práctica, partiendo desde la problemática que se presenta en el contexto.

Lo planteado, también tiene su respuesta en el Proyecto Tuning Europa (González y Wagenaar 2003), donde las competencias son entendidas como una combinación dinámica de atributos en relación a conocimientos, habilidades, comprensión y capacidades, que describen los resultados del aprendizaje de un programa educativo. El constructo competencias está ligada a su vez a la elección de una forma de homologar las titulaciones que expiden cada una de las universidades, especificando el perfil que ha logrado el egresado (Gimeno, 2008), las competencias por tanto no son aspiraciones, buenos deseos o metas, sino son las destrezas que se pueden observar, medir y demostrar (Villanueva, 2010).

El desafío para el sistema universitario en pleno milenio no solo se limita a la formación por competencias sino al desarrollo de las competencias tecnológicas, considerando que, desde las instituciones educativas, se debe aprovechar las tecnologías para investigar, conocer, diagnosticar y aprender del uso y manejo de los mismos. Según la Organización para la Cooperación y el Desarrollo Económico (OCDE, 2002), con la globalización y la modernización se ha venido creando un mundo cada vez más interconectado. Para comprender y funcionar bien en este mundo, las personas necesitan, dominar las tecnologías cambiantes y comprender enormes cantidades de información 
que se encuentra disponible en la red, tecnologías que facilitan al docente, así como también al estudiante en los procesos de comunicación, investigación y obtención de información oportuna.

Con referencia a las Tecnologías de Información y Comunicación (TIC) y con relación a su potencial para mejorar la educación, según Coll (2007) solo mediante la transformación del contexto de uso de las TIC se podrá generar un impacto considerable dirigido a innovar la enseñanza y por ende mejorar el aprendizaje. Con esa incidencia favorable en al ámbito educativo las TIC vienen siendo hoy en día una de las herramientas que ha incursionado velozmente en la educación.

Dentro del contexto latinoamericano, la educación basada en competencias se viene introduciendo de manera exitosa en las diferentes universidades, mientras que con referencia a las TIC se vienen estableciendo Planes de Acción Regional para América Latina y el Caribe por medio de las conferencias ministeriales para la sociedad de la información (eLAC), en la que se conciben las TIC como instrumentos de desarrollo económico y de la inclusión social (Sunkel y Trucco, 2013). Sin embargo, en Bolivia se evidencia cierto rezago en cuanto a la aplicación de la educación basada en competencias, ante la ausencia de planes de acción provenientes del ministerio de educación, donde si bien existen bases normativas para su implementación como la la Ley 070 del 2010, que asume un conjunto de reformas que abarca desde la educación básica, pasando por la educación media hasta llegar a la educación superior, dejando de lado la formación enciclopédica, y centrado en la enseñanza que prioriza contenidos disciplinares, por un modelo curricular "flexible" plantea el modelo de formación por competencias, como uno de sus atributos más distintivos. Y con referencia al desarrollo de las competencias tecnológicas hacen falta planes de acción educativos que permitan la reestructuración desde los enfoques pedagógicos, reglamentos, diseños y contenidos curriculares acorde a las innovaciones tecnológicas que se presentan hoy en día.

Por tanto, en correspondencia con lo expresado anteriormente el presente trabajo se ha centrado en la revisión sistemática de la literatura sobre competencias y competencias tecnológicas y se planteó como objetivo: preponderar la importancia del enfoque de formación por competencias, así como el desarrollo de las competencias tecnológicas generando lineamientos/pautas que permitan su aplicación en la formación superior en Bolivia.

En definitiva, el enfoque basado en competencias nace no solo de la necesidad de formar profesionales competentes que respondan adecuadamente a las necesidades sociales y del trabajo, que ahora son tan valoradas; sino que implica también una formación superior, centrada en la persona, esto significa un proceso dinámico en donde el estudiante tiene una participación más activa en su aprendizaje, situación que se complementa con la incursión de las TIC al campo educativo donde los estudiantes presentan una mejor asimilación hacia el aprendizaje y un mejor manejo de las mismas, mientras que el docente puede hacer uso de las tecnologías informáticas y otros recursos en aula, sin embargo esto requiere de un uso y manejo adecuado de tales medios (Navia, 2018).

Estos dos procesos de transformación como son la educación por competencias y el desarrollo de las competencias tecnológicas, si son adecuadamente implementadas y conducidas buscan contribuir en el sistema universitario boliviano, la calidad educativa, así como en la satisfacción de las diferentes necesidades y demandas sociales.

\section{MÉTODO}

La revisión sistemática de la literatura viene a ser un medio para poder identificar, seleccionar evaluar e interpretar críticamente todas las investigaciones relevantes que se encuentran disponibles. Las 
revisiones sistemáticas se caracterizan por tener y describir el proceso de elaboración transparente y comprensible para poder recolectar, seleccionar, evaluar críticamente y resumir toda la evidencia disponible (Letelier, Manríquez y Rada, 2005). El proceso de revisión sistemática se realizó con base a la metodología propuesta por Moreno et al (2018) en la cual plantea y recomienda las siguientes fases: 1) Planteamiento de la pregunta estructurada, 2) Búsqueda en base de datos, 3) Selección de artículos, 4) Extracción de datos y 5) Análisis.

\section{Planteamiento de la pregunta estructurada}

Esta fase del proceso de revisión bibliográfica inició con la formulación de la(s) pregunta(s) de investigación. Acorde al objetivo propuesto que busca preponderar la importancia del enfoque de formación por competencias, así como el desarrollo de las competencias tecnológicas generando lineamientos/pautas que permitan su aplicación en la formación superior en Bolivia. Para ello se definieron las siguientes preguntas de investigación:

1. ¿Cuál la importancia de la formación por competencias en la educación superior?

2. ¿Cuál la importancia del desarrollo de las competencias tecnológicas?

\section{Búsqueda en base de datos}

A partir de las dos preguntas orientadoras, se delimitaron los descriptores: competencias, competencias tecnológicas y educación superior. Fueron aplicadas combinaciones entre tales descriptores: Competencias en la educación superior; competencias tecnológicas en la educación superior.

Las bases de datos que se exploraron y analizaron incluyeron: revistas electrónicas (Dialnet, Redalyc, Scielo, Google académico, entre otras), así como documentos, libros e informes escritos sobre el tema. Teniendo acceso a una información de contenido significativo para responder a los niveles del conocimiento científico que identifica un estudio de revisión documental.

\section{3) Selección de artículos}

Para la selección de la muestra en la literatura y selección de datos, se asumieron los siguientes criterios de inclusión: artículos científicos de abordaje metodológico diverso sin límite en la fecha de antigüedad, disponibles en el formato texto completos y de acceso libre a los investigadores, que guarden relación con la temática en estudio. Artículos publicados en alguna revista de investigación nacional o internacional, en idiomas español, portugués e inglés también fueron incluidos. La selección de los artículos incluyó una evaluación inicial por medio de los títulos, resúmenes y palabras clave, cumplidas las exigencias, la lectura y análisis eran realizados en su totalidad. Se excluyeron los artículos donde solamente estaban disponibles los resúmenes, que presentaran duplicidad y las publicaciones que no se relacionaban a la temática de estudio.

La revisión proporcionó un total de 145 resultados, y de estos fueron seleccionados 30 artículos. Cabe señalar que el número de material excluido (115) se debe a que no respondían a los criterios de inclusión establecidos, muchos artículos no estaban disponibles para acceso libre o presentaron duplicidad. Se procuró por tanto complementar la información con documentos disponibles en cuanto a informes emitidos como ser: la UNESCO, CEUB, AGETIC, OCDE y otros procurando mantener el rigor científico del presente estudio.

\section{4) Extracción de datos}

Esta etapa permitió la revisión sistemática de la literatura, la selección de los estudios primarios, ver la relevancia y credibilidad de lo sustentado por 
cada uno de los autores para su posterior análisis; lo cual requirió una lectura total de los artículos seleccionados.

\section{5) Análisis}

Etapa que permitió realizar un análisis pormenorizado de las investigaciones seleccionadas y la realización de la síntesis de los datos obtenidos.

\section{DESARROLLO Y DISCUSIÓN}

A continuación, se presentan algunos resultados emergentes de la revisión bibliográfica, partiendo de algunos elementos teóricos consistentes sobre competencias, que denotan su carácter polisémico y como es que a partir de este enfoque la educación se centra en el estudiante, al ser este el directo responsable de construir sus propios conocimientos y ponerlos en práctica. Así también se abordó sobre las competencias tecnológicas desde los elementos teóricos que la sustentan y su incidencia en la formación desde la incursión de las TIC en la educación superior, planteando algunas pautas producto de la revisión realizada.

\section{Competencia}

Desde la Organización para la cooperación y el Desarrollo Económicos (OCDE, 2002) define las competencias como las habilidades que permiten cumplir con éxito todas y cada una de las exigencias mediante la movilización de los prerrequisitos que la sociedad otorga. Para Perrenaud (2006) las competencias responden a la capacidad de poder actuar de una manera eficaz en una determinada situación.

Mientras que según Posada Álvarez (2004) el concepto mas generalizado sobre competencia es el de "Saber hacer en un contexto", el mismo que requiere de conocimiento (teórico, práctico o teórico práctico), mismos que se expresan en el desempeño al ejecutar una acción en un determinado entorno. Tobón (2006) establece que las competencias vienen a ser los procesos complejos de desempeño de manera idónea, al igual que posada, señala que dicho desempeño debe realizarse en un determinado contexto de una manera responsable. Para Mastache (2007) las competencias:

Permiten que las personas resuelvan
problemas y realicen actividades
propias de su contexto profesional
para cumplir con los objetivos o
niveles preestablecidos, teniendo en
cuenta la complejidad de la situación
y los valores y criterios profesionales
adecuados, mediante la articulación
de todos los saberes requeridos (p. 40 ).

Al definir competencia para Benarroch (2015) más alla de su carácter polisémico, lo considera como una acción exitosa o efectiva que tiene una persona para poder desarrollar una actividad en cualquier tipo de situación dentro de un ámbito concreto. Cualidad que puede precisarse con tres observables:

- Repetición: ante situaciones semejantes la reconocida competencia del sujeto se ejecuta con resultados parecidos una y otra vez (variación intracontextual).

- Generalización: ante la diversidad de variaciones del contexto en el escenario donde se ubica la competencia, el sujeto que la posee sabe responder con resultado también exitoso (variación intercontextual).

- Diferenciación: se refiere a la adecuación o acomodación de la competencia ante situaciones o circunstancias novedosas que de pronto aparecen en el escenario práctico donde se da el actuar competente del sujeto (p. 11). 
En América Latina la incorporación del enfoque de competencias se vinculó sobre todo a las políticas de empleo y se abrió paso sobre todo a partir de la educación media técnica y en la formación superior (Mastache, 2007). Dentro del proyecto Tuning América Latina (2007):

La competencia, en educación, se presenta como una red conceptual amplia, que hace referencia a una formación integral del ciudadano, por medio de nuevos enfoques, como el aprendizaje significativo, en diversas áreas: cognoscitiva (saber), psicomotora (saber hacer, aptitudes), afectiva (saber ser, actitudes y valores). En este sentido, la competencia no se reduce al simple desempeño laboral, tampoco a la sola apropiación de conocimientos para saber hacer, sino que abarca un conjunto de capacidades que se desarrollan a través de procesos que conducen a la persona responsable a ser competente para realizar múltiples acciones... por las cuales uno proyecta y evidencia su capacidad de resolver un problema dado, dentro de un contexto específico y cambiante (p. 36).

Se puede concebir que la competencia es la capacidad de adquirir habilidades y destrezas que permiten el desarrollo de acciones en un determinado contexto, bajo el principio de teoríapráctica, siendo que es en este proceso que se vincula el contenido científico, con el investigativo y el productivo. En esta lógica, las escuelas tienen como una de sus funciones, no solo al de contribuir al desarrollo integral de la persona, sino también el de formar ciudadanos que puedan insertarse en la vida productiva, por tanto, la escuela debe asumir el compromiso de poder crear las condiciones necesarias para que los estudiantes tengan la posibilidad de obtener un empleo (Trujillo-
Segoviano, 2014) más aun en un entorno global y dinámico que caracteriza a los últimos tiempos.

Bolivia reconoce la formación con enfoque basado de competencias desde la promulgación de la Constitución Política del estado (CPE, 2009) el artículo 91, señala: "la educación superior es intercultural y plurilingüe, y tiene por misión la formación integral de recursos Humanos con alta calidad y competencia profesional...” (p. 22). En tanto que la Ley 070 (2010), el artículo 53 establece que la formación superior tiene como uno de sus objetivos:

Formar profesionales científicos,
productivos y críticos que garanticen
un desarrollo humano integral,
capaces de articular la ciencia y
la tecnología universal con los
conocimientos y saberes locales que
contribuyan al mejoramiento de la
producción intelectual, y producción
de bienes y servicios, de acuerdo con
las necesidades presentes y futuras
de la sociedad y la planificación del
Estado Plurinacional (p. 23).

Desde el Comité Ejecutivo de la Universidad Boliviana (CEUB, 2014), dentro de su informe presentado en la I-XII Reunión Académica Nacional, asume como una de sus políticas académicas el diseño curricular con el enfoque de formación basada en competencias a nivel de todas las universidades que conforman esta institución, y define las competencias como:

Un proceso complejo de desempeño con idoneidad y responsabilidad social, que moviliza, combina y transfiere con efectividad un conjunto integrado de recursos internos (conocimientos, habilidades, actitudes) y externos (información, recursos tecnológicos y otros) para resolver problemas en la vida cotidiana, laboral-profesional 
en un contexto intercultural y pluralismo epistémico, aportando a la construcción y transformación de la realidad (p. 37).

En este sentido por los planteamientos que se realiza desde la Ley de educación en Bolivia, la CPE asi como la CEUB, la formación por competencias se centra en el aprendizaje lo cual implica darle un protagonismo activo al estudiante, por lo que se considera como un paradigma que está sustentado principalmente en diversas corrientes psicológicas constructivistas y que han propugnado por desarrollar habilidades y capacidades totales en el individuo es desde ahí que surgen las tendencias en el desarrollo de este enfoque no solo desde el conductismo, el constructivismo o el humanístico -crítico- sino tambien desde lo holístico (TrujilloSegoviano, 2014). Donde lo importante no es la producción de conocimiento, sino cuánto sabe el estudiante reproducir lo que le han enseñado, ser el responsable de gestionar y evidenciar sus aprendizajes y ponerlo en práctica para demostrar su dominio de ese saber.

Para desarrollar las competencias desde el ámbito educativo, es imprescindible conocer las demandas del mercado laboral, así como las exigencias del mundo actual. Irigoin (1998) enuncia que una persona competente para el trabajo deberá adquirir conocimientos, desarrollar un sinfín de habilidades, necesitando al mismo tiempo el poder desarrollar actitudes y habilidades que le permitan tomar decisiones en la resolución de problemas y de conflictos que se presenten en su fuente laboral.

Con la revisión teórica y normativas nacionales de Bolivia se plantea que las competencias en sí, son diseños complejos, de carácter polisémico e integrales donde para el logro de las competencias es elemental la utilización de conocimientos, habilidades y valores de una forma articulada, que permita dar soluciones a los futuros profesionales a cada una de las problemáticas que se presentan en un contexto determinado, desde esta percepción radica la importancia en asumir este enfoque de formación basada en competencias dentro de la educación superior.

\section{Competencias tecnológicas}

De la revisión bibliográfica se obtuvieron los siguientes resultados.

La tendencia de la educación superior pos y transmoderna tiene como tarea la implementación de las TIC en las aulas de clase, considerando que hoy en día los estudiantes tienen facilidades para acceder a la información y a la tecnología situación que les permite poder educarse e informarse, en un mundo donde las herramientas tecnológicas y los medios digitales ocupan un lugar significativo tanto fuera como dentro de las aulas (Novillo et al., 2017). Para Almerich et al (2010) las competencias tecnológicas son la capacidad para seleccionar $\mathrm{y}$ utilizar de forma pertinente, responsable y eficiente una variedad de herramientas tecnológicas entendiendo los principios que las rigen, la forma de combinarlas y su utilización en el contexto académico. Estas competencias giran en torno a capacidades como la búsqueda de información, aplicación en el trabajo-producción, comunicación, gestión de la información, colaboración y aspectos éticos.

Desde la UNESCO (2019) las competencias digitales son un cúmulo de competencias que vienen a facilitar el uso de los dispositivos digitales, las aplicaciones de la comunicación, así como las redes que van a permitir el acceso a la información. Competencias que a su vez permiten crear e intercambiar contenidos digitales, comunicar y colaborar, asimismo dar soluciones problemas como ser el uso de procesadores de texto y programas de presentación, aplicaciones de correo electrónico y redes sociales. Gracias a la utilización permanente de las TIC en el proceso enseñanza aprendizaje, 
los estudiantes pueden desarrollar diferentes capacidades, habilidades en cuanto al uso y manejo de las TIC. Dentro de un contexto educativo sólido, desde la UNESCO (2019) la tecnología puede hacer que los estudiantes adquieran a su vez las capacidades para:

- utilizar las tecnologías de la información;

- buscar, analizar y evaluar información;

- resolver problemas y elaborar decisiones;

- utilizar instrumentos de producción con creatividad y eficacia;

- comunicar, colaborar, publicar y producir; y

- ser ciudadanos informados, responsables y capaces de aportar contribuciones a la sociedad (p. 3).

Es incuestionable la incorporación de las TIC en las universidades siendo que permite el desarrollo de capacidades como los señalados por la UNESCO en los estudiantes y a su vez una serie de habilidades en el plantel docente. Por lo que es necesario una ubicación de la responsabilidad de los recursos de las TIC para la docencia y para el proceso de enseñanza y aprendizaje (Domínguez Díaz, 2016).

Desde la Comisión Europea y su plan de acción de educación digital (2021-2027) se pretende fomentar la alfabetización digital, con la finalidad de desarrollar capacidades y competencias digitales básicas desde edades tempranas, así como competencias avanzadas que generen más especialistas digitales. Mientras que, en la mayoría de los países de América Latina y el Caribe, se viene desarrollando algunas políticas de TIC en el sector de la educación donde se plantean varias metas como las de generar innovación, el cambio en las prácticas de enseñanza y aprendizaje y el perfeccionamiento profesional de los docentes (Sunkel et al, 2014). Metas que por lo señalado buscan el desafío de mejorar la calidad de la educación.

La CEUB (2014) recomienda impulsar y fortalecer las TIC manifestando que los mismos posibilitan de sobremanera mejorar el proceso de desarrollo de la formación basada en competencias. El Comité plurinacional de Tecnologías de la Información y comunicación (COPLUTIC) tiene elaborado un plan de implementación (2017-2025) donde establece que desde las tecnologías de la información y comunicación se puede consolidar la educación (AGETIC, 2017). Asimismo, en los últimos años Bolivia ha desarrollado diversas políticas públicas que van desde la ampliación de la cobertura de internet en todo el territorio nacional, hasta el uso de TIC en el ámbito educativo, ampliando los alcances de la alfabetización digital y el empoderamiento de la población en el uso de estas tecnologías (AGETIC, 2019).

El modo en que la tecnología se integra en las actividades de enseñanza y aprendizaje es crucial para mejorar los resultados de los estudiantes, donde el uso de la tecnología debe ser la herramienta que permita impulsar pedagogías más innovadoras donde los docentes puedan fomentar el aprendizaje de los estudiantes por medio del uso de las TIC (OCDE, 2020), considerando que las funcionalidades y beneficios de las TIC dentro del campo de la educación, varía dependiendo al autor, Baelo y Cantón (2009) sintetiza y señala que entre los principales beneficios de la inclusión de las TIC en la educación superior se tiene:

- La facilidad para el acceso a la información y la variedad de información disponible,

- Los elevados parámetros de fiabilidad y rapidez de que disponen para el procesamiento de la información y los datos, 
- La variedad de canales de comunicación que permiten,

- La eliminación de las barreras espaciotemporales,

- Las posibilidades de feek-back que ofertan,

- El desarrollo de espacios flexibles para el aprendizaje,

- La potenciación de la autonomía personal y el desarrollo del trabajo colaborativo,

- La optimización de la organización y el desarrollo de las actividades docentes e investigadoras,

- La agilización de las actividades administrativas y de gestión, además de permitir su deslocalización del contexto inmediato (p. 3).

Por cuanto las TIC se han convertido hoy en día en elementos esenciales no solo para la actividad docente sino también para la interacción del estudiante, donde las competencias tecnológicas, no solo proporcionan la capacidad de aprovechar la riqueza de las nuevas posibilidades asociadas con las tecnologías digitales y los retos que plantean, sino que resulta cada vez más necesaria para poder participar de forma sistemática en la nueva sociedad y economía del conocimiento del siglo XXI (INTEF, 2017).

Se hace necesario por tanto, que dentro de las instituciones universitarias, se asuma una serie de medidas donde desde el Instituto Nacional de Tecnologías Educativas y de Formación del profesorado (INTEF, 2017) medidas tales como: la capacitación al plantel docente para una plena integración de los medios tecnológicos, buscar y probar nuevas aplicaciones y herramientas de comunicación en línea siempre con el objetivo y la motivación de mejorar el proceso enseñanza aprendizaje y otra medida quizá la más importante invertir en la adquisición de medios tecnológicos de calidad, así como en infraestructura para brindar las condiciones necesarias que permita al estudiante como al docente aplicar adecuadamente tales medios.

Tomando en cuenta a su vez, que para ser un competente digital los estudiantes deben tener un dominio estratégico de cinco grandes capacidades asociadas respectivamente a las diferentes dimensiones compuestas a su vez de cinco indicadores planteado según Marqués, 2009 citado por Amor y Delgado (2012):

- La dimensión del aprendizaje (supone la transformación de la información en conocimiento y su adquisición),

- La dimensión informacional (supone la obtención, la evaluación y el tratamiento de la información en entornos digitales),

- La dimensión comunicativa (abarca la comunicación interpersonal y la social),

- La dimensión de la cultura digital (abarca las prácticas sociales y culturales de la sociedad del conocimiento y la ciudadanía digital),

- La dimensión tecnológica (abarca la alfabetización tecnológica y el conocimiento y dominio de los entornos digitales) (pp. $30,31)$.

En este sentido serán los docentes los encargados de promover y desarrollar en los estudiantes estas capacidades en cuanto a conocimientos, habilidades, valores y actitudes necesarios en cuanto a TICs.

Mientras que para ser un competente digital desde el rol docente, la UNESCO (2007), los organiza según tres enfoques didácticos diferentes: 
- Noción básica de TIC; el objetivo de este enfoque es preparar estudiantes, ciudadanos y trabajadores capaces de comprender las tecnologías para apoyar el desarrollo social.

- Profundizacion del conocimiento; consiste en aumentar la capacidad de los educandos, los ciudadanos y la mano de obra para añadir valor a la sociedad y la economía, aplicando los conocimientos adquiridos en su formacion, con vistas a resolver problemas complejos encontrados en situaciones reales de la vida laboral y diaria.

- Generación de conocimiento; consiste en aumentar la participacion cívica, la creatividad cultural y la productividad económica, formando estudiantes, dedicados a la tarea de crear conocimientos, innovar y participar en la sociedad del conocimiento (pp. 11-14).

Las competencias digitales se han asumido como una combinación de conocimientos, habilidades y capacidades, en conjunción con los valores y actitudes, que permitan alcanzar los objetivos de manera eficaz y eficiente en contexto y con herramientas digitales (Amor y Delgado, 2012), permitiendo a docentes ser hoy en día una de las herramientas más empleadas en aula y a los estudiantes permitiéndoles ser más críticos con toda la información que obtienen. La incidencia de las TIC en la formación superior más allá de ser una herramienta con muchos beneficios, aporta en aspectos trascendentales en aula como la motivación, el interés, la interactividad, el trabajo colaborativo, así como independiente, desarrollo de la creatividad, la comunicación y la actualización en tiempo real.
Por tanto, las competencias, así como las competencias tecnológicas en la educación superior han permitido el desarrollo de un nivel completo de competencias tanto cognitivas, afectivas, conductuales, así como las competencias digitales contribuyendo a un aprendizaje integral y significativo dentro de la formación de los estudiantes.

\section{Discusión}

Según el objetivo planteado el artículo abarcó dos elementos: por una parte, el referido a remarcar la importancia del enfoque de formación por competencias, y por otra el desarrollo de las competencias tecnológicas en la educación superior.

Respecto al primer elemento, se puede señalar en principio que se tiene claro que el enfoque educativo actual asumido en la formación superior es el enfoque basado en competencias, esto porque, tiende a una educación centrada en el estudiante, lo que implica una participación más activa en su aprendizaje y donde se promueve el trabajo en equipo a través de su interacción con otros estudiantes, entonces, los protagonistas (docente-estudiante) asumen roles diferentes a los del modelo educativo tradicional, el uso de la tecnología en su formación profesional dentro de un modelo constructivista que permite al estudiante desarrollar habilidades para el autoaprendizaje, el trabajo colaborativo, la responsabilidad en la toma de decisiones entre otros aspectos; en tanto que el docente se convierte en guía y mediador del conocimiento, asegurándose del aprendizaje significativo de los estudiantes. En un entorno constructivista, el aprendizaje es esencialmente activo y dinámico, a través del cual el estudiante construye sus propios conocimientos a partir de su experiencia e integrándola con la información que recibe (Arreola et al, 2016, citado por Navia Pérez, 2018). Un modelo educativo que a su vez demanda la integración de las TIC tanto a la práctica docente como a la práctica del estudiante. 
El enfoque de competencias tiene ecos halagadores para la formación de las jóvenes generaciones, así como la indiscutible vinculación que se da entre la teoría-práctica, la integración de todas y cada una de las áreas básicas, profesionales y complementarias, un trabajo interdisciplinario, colocando al estudiante frente a situaciones reales dentro de su futuro ejercicio profesional para que vaya adquiriendo capacidades que le permitan asumir responsabilidades una vez obtenga el título en sus manos, y pueda desde ese momento cumplir con los requerimientos sociales y de las escuelas universitarias (Camperos, 2008).

Respecto al segundo elemento, referida al desarrollo de competencias tecnológicas, es preciso quelos docentes, así como los estudiantes desarrollen una serie de conocimientos, habilidades y actitudes relacionados con las TIC, para aprovechar al máximo las posibilidades que brindan (Hernández e Iglesias, 2017). Esta afirmación es corroborada por otros autores, como Gros, Silva, Garrido, y Rodríguez quienes plantearon que: los docentes deben demostrar un dominio de las competencias asociadas al conocimiento general de las TIC y al manejo de las herramientas de productividad (procesador de texto, hoja de cálculo, presentador) e Internet, desarrollando habilidades y destrezas para el aprendizaje permanente de nuevos hardware y software y los estudiantes deben poseer las capacidades necesarias en el uso de las tecnologías en sus clases, si bien la mayoría de ellos las maneja, les faltan habilidades para su uso en clases (Garrido et al, 2006). En correspondencia encontramos que para su desarrollo es importante asumir las medidas necesarias como la actualización tecnológica, el desarrollo de nuevas estrategias, capacitaciones oportunas sobre las TIC, entre otras.

Comparando las competencias tecnológicas que debe reunir el estudiante, así como el docente, se puede señalar que ambos deben reunir las capacidades necesarias para el uso de redes, internet, entornos virtuales, entre otros; que propicien el desarrollo en cuanto el uso de herramientas digitales. La educación superior ya ha dado un paso muy importante con los avances e implementación de la tecnología en aula, ahora depende de los actores directos explotar los beneficios que esto genera en el ámbito de la educación.

\section{CONCLUSIONES}

Se debe comprender que el enfoque de formación basado en competencias tiene un carácter polisémico, de uso variado, con múltiples interpretaciones, características propias y asumida desde diferentes perspectivas según los autores consultados, este enfoque viene a ser hoy en día uno de los más asumidos por las diferentes universidades del sistema para promover el desarrollo de competencias centrado en el estudiante y contextualizando las especificidades del mismo en el ámbito laboral y contextual.

Dentro de las competencias profesionales que adquiera el estudiante tales como los conocimientos, habilidades y actitudes, se constituyen en una alternativa para su formación integral, con características técnico pedagógicas propias desarrollando competencias laborales propias con criterios de calidad.

Es necesario que las instituciones de formación superior tomen en cuenta las diferentes medidas que se deben tomar en cuenta para la implementación adecuada de las TIC como son las capacitaciones, la búsqueda de herramientas y el equipamiento de elementos necesarios, si lo que se pretende, es avanzar hacia el conocimiento universal a través del uso de los medios tecnológicos.

Los resultados obtenidos permiten a su vez, servir de base para asumir políticas educativas pertinentes, en la realización de programas de formación y cursos centrados en las competencias digitales, para mejorar la integración de las TIC en la educación superior, siendo que por lo 
señalado son los estudiantes los agentes activos en el proceso enseñanza aprendizaje, y los docentes los mediadores del mismo, y conseguir el desarrollo de las competencias tecnológicas en ambos actores, va a contribuir en un cambio trascendental en la calidad académica. Más aun considerando a las universidades como los espacios donde se deben desarrollar las competencias profesionales necesarias en sus estudiantes como las de investigación, criticidad, trabajo en equipo y competencias tecnológicas en una sociedad cambiante donde los futuros profesionales serán su fuente de productividad e innovación. Por otro lado, se espera que, con el desarrollo de las competencias tecnológicas se contribuya al mejoramiento de la calidad educativa y la dinamización del proceso enseñanza aprendizaje.

$\mathrm{Si}$ antes las universidades del sistema universitario boliviano no entregaban resultados a la sociedad sin mayores exigencias que la formación profesional en un plazo pertinente; con la implementación del enfoque de formación basada en competencias respaldada desde normativas nacionales, se puede exigir como sociedad con mayor rigor y juicio autocrítico; la formación de profesionales con capacidad, eficiencia, eficacia y competitividad.

\section{REFERENCIAS}

AGETIC. (2017). Plan de implementación de gobierno electrónico 2017-2025. https:// coplutic.gob.bo/IMG/pdf/plan_gobierno_ electronico_.pdf

AGETIC. (2019). Juventudes TIC. Estudios sobre las TIC en adolescentes y jóvenes de Bolivia. Quatro Hermanos. https://www.agetic.gob.bo/ pdf/estadotic/libro_juventudes_tic.pdf

Almerich, G., Suarez, J. M., Orellana, N. y Díaz, M.. (2010). La relación entre la integración de las tecnologías de la información y comunicación y su conocimiento. Revista De Investigación Educativa, 28(1), 31-50. https://revistas.um.es/ rie/article/view/97861
Amor, M., y Delgado, A. (2012). De la competencia digital y audiovisual a la competencia mediática: dimensiones e indicadores. Comunicar, $\mathrm{xx}(39)$, 25-34. https://www.redalyc.org/articulo. oa? $\mathrm{id}=15823945003$

Armengol, C., Meneses, J. y Rodríguez, D. (2017). La adquisición de las competencias profesionales a través de prácticas curriculares de la formación inicial de maestros. Revista de Educación(376), 229-251. https://doi:10.4438/1988-592XRE-2017-376-350

Baelo, R. y Cantón, I. (2009). Las tecnologías de la información y la comunicación en la educación superior. Estudio descriptivo y de revisión. Revista Iberoamericana de Educación(50), 1-12. http://hdl.handle.net/10612/12086

Benarroch, A. y Nuñez, G. (2015). Aprendizaje de competencias científicas versus aprendizaje de contenidos específicos. Una propuesta de evaluación. Enseñanza de las ciencias, 33(2), 9 27. doi: $10.5565 / \mathrm{rev} /$ ensciencias. 1578

Camperos Camero, M. (2008). Evaluation based on competences, myths, dangers and challenges. Educere, 12(43), 805-814. http://ve.scielo.org/ scielo.php? script $=$ sci_arttext $\&$ pid $=S 1316$ 49102008000400017\&lng=es\&tlng=en

CEUB. (2014). I- XII Reunión Académica Nacional, Informes, documentos y resoluciones. http:// www.ceub.edu.bo/academica/documentos/ ranes/15_I_XII_RAN_UAP_14.pdf

Coll, C. (2008). Aprender y enseñar con las TIC: expectativas, realidad y potencialidades. En R. Carneiro, J.C. Toscano y T. Díaz. (Eds.) Los desafíos de las TIC para el cambio educativo (pp. 113-126). Santillana

Corvalán, O. (2008). Las competencias ya no son lo que eran (ni serán lo que son). Revista Electrónica de desarrollo de competencias, 1(1), 10-30.

Constitución Política del Estado Plurinacional de Bolivia. (2009, 7 de febrero). Congreso del Estado Plurinacional de Bolivia. www. gacetaoficialdebolivia.gob.bo 
Domínguez Díaz, Y. (2016). La utilización de las Tecnologías de la información y las comunicaciones (TIC) en el aprendizaje universitario. Revista Universidad y Sociedad, 8(4), 158-163. http://scielo.sld.cu/ scielo.php?script $=$ sci_arttext\&pid $=$ S22 18 $36202016000400021 \& \operatorname{lng}=$ es\&tlng=es

Comisión Europea (2021). Web oficial de la Unión Europea. https://ec.europa.eu/education/ education-in-the-eu/digital-education-actionplan_es

Garrido, J., Gros, B., Rodríguez, J. y Silva, J. (2006). Estándares en Tecnología de la Información y la Comunicación para la Formación Inicial Docente: Situación actual y el caso chileno. Revista Iberoamericana de Educación, 38(1), 51-68. https://www.researchgate.net/profile/ Jose-Garrido

Miranda/publication/28106773_Estandares_ en_tecnologias_de_la_informacion_y_la_ comunicacion_para_la_formacion_inicial_ docente_situacion_actual_y_el_caso_chileno/ links/0fcfd51433d0a35ba1000000/Estandaresen-

Gimeno, J., Pérez, A., Bautista, J., Torres, J., Angulo, F. y Álvarez, J. (2008). Educar por competencias, ¿qué hay de nuevo?. MORATA S.L. https:// books.google.com.bo/books?id=iZkjEAAA QBAJ\&printsec $=$ frontcover \&dq $=$ Educar + po $\mathrm{r}+$ competencias, $+\% \mathrm{C} 2 \% \mathrm{BFqu} \% \mathrm{C} 3 \% \mathrm{~A} 9+\mathrm{ha}$ $\mathrm{y}+\mathrm{de}+$ nuevo $\% 3 \mathrm{~F} \& \mathrm{hl}=\mathrm{es}-419 \& \mathrm{sa}=\mathrm{X} \&$ redir e $s \mathrm{c}=\mathrm{y} \# \mathrm{v}=\mathrm{o}$ e e p a g $\& \mathrm{q}=\mathrm{Edu} \mathrm{c}$ a $\mathrm{r} \% 20$ p or $\% 20$ competencia s\% 2 C $\% 20$ $\%$ C $2 \%$ B Fqu \% C 3\% A 9\% 20 hay $\% 20$ de $\% 20$ nuevo $\% 3 \mathrm{~F} \& \mathrm{f}=$ false

González, J. y Wagenaar, R. (Eds.) (2003). Tuning Educational Structures in Europe. Informe Final. Universidad de Deusto. http://tuningacademy. org/wp-content/uploads/2014/02/TuningEUI_ Final-Report_SP.pdf

Hernández, A. e Iglesias, A. (2017). La importancia de las competencias digitales e informacionales para el desarrollo de una escuela intercultural. INTERACÇÕES, 13(43), 205-232. https://doi. org/10.25755/int.12038
Instituto Nacional de Tecnologías Educativas y de formación del Porfesorado. (2017). Marco común decompetencia digital docente.Minsterio de educación cultura y deporte de España. http://educalab.es/documents/10180/12809/ MarcoComunCompeDigiDoceV2.pdf/ e8766a69-d9ba-43f2-afe9-f526f0b34859

Irigoin, M. (1998). En torno al concepto de competencias. En L. Hidalgo. (Comp.) Gestión de la capacitación en las organizaciones. Conceptos básicos. (pp. 43-47). Ministerio de Salud

Letelier, L., Manríquez, J y Rada, G. (2005). Revisiones sistemáticas y metaanálisis: ¿son la mejor evidencia? Revista médica de Chile, 133(2), 246-249. https://dx.doi.org/10.4067/ S0034-98872005000200015

Ley $\mathrm{N}^{\circ} 070$ de 2010. (2010, 20 de diciembre). Congreso del Estado Plurinacional de Bolivia. www.gacetaoficialdebolivia.gob.bo

Mastache, A. (2007). (1ra). Aclaraciones y conceptualizaciones. En A. Mastache, D. Miguez, L. Nantes, M. Cedrato, M. Orlando y M. Kurlat. (Eds.) Formar personas competentes. (pp. 23-56 ). Novedades Educativas

Moreno, B., Muñoz, M., Cuellar, J., Dmancic, S. y Villanueva, J. (2018). Revisiones Sistemáticas: definición y nociones básicas. Revista clínica de periodoncia, implantología y rehabilitación oral, 11(3), 184-186. https://dx.doi.org/10.4067/ S0719-01072018000300184

Navia PérezJ. (2018). La tarea educativa frente a las transformaciones tecnoógicas. Educación Superior CEPIES, 4(1), 41-53 http:// www.scielo.org.bo/scielo.php? script $=$ sci_ arttext\&pid=S2518-82832018000100008\&lng= es\&tlng=es

Novillo, E., Espinoza, M. y Guerrero, R. (2017). Influencia de las TIC en la educación universitaria, caso Universidad Técnica de Machala. Dialnet, 2(3), 69-79 
OCDE. (2002). La definición y selección de competencias clave. Publicaciones de la OCDE https://www.deseco.ch/bfs/ deseco/en/index/03/02.parsys.78532. downloadList.94248.DownloadFile.tmp/2005. dscexecutivesummary.sp.pdf

OCDE. (2020). Aprovechar al máximo la tecnología para el aprendizaje y la formación en América Latina. Publicaciones de la OCDE. https://www. oecd.org/skills/centre-for-skills/Aprovechar_ al_m\%C3\%A1ximo_la_tecnolog\%C3\%ADa_ para_el_aprendizaje_y_la_formaci\%C3\%B3n_ en_Am\%C3\%A9rica_Latina.pdf

Perrenaud, P. (2006). Construir competencias desde la escuela. Noreste LTDA

Posada Álvarez, R. (2004). Formación superior basada en competencias, interdisciplinariedad y trabajo autónomo del estudiante. Revista Iberoamericana de Educación. 35 (1), 1-33. https://doi.org/10.35362/rie3512870

Proyecto Tuning-América Latina 2004-2007 (2007). Reflexiones y perspectivas de la Educación Superior en América Latina. Universidad de Deusto

Sunkel, G., Trucco, D. y Espejo, A. (2014). La integracion de las tecnologías digitales en las escuelas de América Latina y el Caribe. CEPAL

Tobón, S. (2006). Aspectos básicos de la formación basada en competencias. Talca
Trujillo Segoviano, J. (2014). El enfoque en competencias y la mejora de la educación. Ra Ximhai, 10(5), 307-322. https://www.redalyc. org/articulo.oa?id=46132134026

UNESCO. (2007). Normas sobre competencias en TIC para docentes. Organización de las Naciones Unidas para la Educación, la Ciencia y la Cultura. https://www.campuseducacion.com/ blog/wp-content/uploads/2017/02/Normas_ UNESCO_sobre_Competencias_en_TIC_ para_Docentes.pdf

UNESCO. (2019). Marco de competencias de los docentes en materia de TIC UNESCO. Organización de las Naciones Unidas para la Educación, la Ciencia y la Cultura

UNESCO. (2019). Normas sobre competencias en TIC para docentes. Organización de las Naciones Unidas para la Educación, la Ciencia y la Cultura. https://www.campuseducacion. com/blog/wp-content/uploads/2017/02/ Normas_UNESCO_sobre_Competencias_en_ TIC_para_Docentes.pdf

Villanueva, G. y Casas, M. (2010). e-competencias: nuevas habilidades del estudiante en la era de la educación, la globalidad y la generación del conocimiento. Signo y pensamiento, XXIX(56), 124-138. http://www.redalyc.org/articulo. oa? id $=86019348008$ 